\title{
Genome-wide pathway analysis in glioma
}

\author{
Y. H. LEE*, G. G. SONG \\ Division of Rheumatology, Department of Internal Medicine, Korea University College of Medicine, Seoul, Korea \\ *Correspondence: lyhcgh@korea.ac.kr
}

Received April 6, 2014 / Accepted October 14, 2014

\begin{abstract}
The aim of this study was to identify candidate single-nucleotide polymorphisms (SNPs) that may play a role in the susceptibility to glioma, to elucidate their potential mechanisms, and to generate SNP-to-gene-to-pathway hypotheses.

A genome-wide association study (GWAS) dataset of glioma including 509,345 SNPs from 1,856 glioma patients and 4,955 control subjects of European descent was used in this study. Identify candidate Causal SNPs and Pathways (ICSNPathway) analysis was applied to the GWAS dataset.

ICSNPathway analysis identified 6 candidate SNPs, 5 genes, and 9 pathways, which revealed 5 hypothetical biological mechanisms. The hypothetical mechanisms, beginning with the strongest, are summarized as follows: (i) rs667128 alters the role of taste receptor, type 2, member 8 (TAS2R8) in taste receptor activity and taste transduction pathways $(p<0.001$, false discovery rate $(\mathrm{FDR})<0.001 ; p=0.001, \mathrm{FDR}=0.012$, respectively), (ii) rs619381 modulates the effect of taste receptor, type 2 , member 7 (TAS2R7) on taste receptor activity and taste transduction $(p<0.001, \mathrm{FDR}<0.001 ; p=0.001, \mathrm{FDR}=0.012)$, (iii) rs1033583 modulates delta-like protein 1 (DLL1), regulating cell adhesion and segment specification $(p<0.001, \mathrm{FDR}=$ $0.011 ; p=0.001, \mathrm{FDR}=0.032$ ), (iv) rs2232580 affects the role of lipopolysaccharide binding protein $(L B P)$ in the response to lipopolysaccharide, positive regulation of interleukin-6 production, acute inflammatory response, and in macrophage activation $(0.002 \leq p \leq 0.013 ; 0.012 \leq \mathrm{FDR} \leq 0.030)$, and (v) rs4644 and rs4652 regulate lectin, galactoside-binding, soluble, 3 (LGALS3), affecting immunoglobulin binding $(p=0.010$; FDR $=0.040)$.

Using the ICSNPathway to analyze glioma GWAS data, 6 candidate SNPs, 5 genes (TAS2R8, TAS2R7, DLL1, LBP, and LGALS3), and 9 pathways that may contribute to the susceptibility of glioma were identified.
\end{abstract}

Key words: glioma, genome-wide association study, pathway-based analysis

Glioma, a fatal neurological cancer associated with considerable morbidity, represents up $80 \%$ of all malignant brain tumors [1]. Although glioma is a complex and heterogeneous disease and its etiology has not been determined, case-controlled and family studies have established a genetic component to disease susceptibility [2]. Previous genome-wide association studies (GWASs) have uncovered glioma-associated single-nucleotide polymorphisms (SNPs) within genes such as coiled-coil domain containing 26 (CCDC26), regulator of telomere elongation helicase 1 (RTEL1), pleckstrin homology-like domain, family $\mathrm{B}$, member 1 (PHLDB1), tumor protein p53 (TP53), cyclindependent kinase inhibitor $2 \mathrm{~A} / \mathrm{B}(C D K N 2 A / B)$, epidermal growth factor receptor $(E G F R)$, and telomerase reverse transcriptase (TERT) $[2,3]$.

GWASs offer a powerful means to search for genes that confer susceptibility to complex diseases [4]. As a result of increasing numbers of GWASs are being reported, and have led to the discovery and validation of novel disease genes [5]. Although large-scale GWASs have been carried out on complex diseases including glioma, many genetic components that contribute to variations in glioma remain unexplained.

Reports to date suggest that individual genes and genetic variants interact with each other to confer small risk contributions to glioma susceptibility. Although a number of genetic signals have been examined at the single-marker level in glioma GWAS studies, the biological mechanisms identified remain controversial [6]. One of the key challenges in interpreting GWAS data is to identify glioma-associated SNPs and provide evidence for the hypothetical mechanisms that could be responsible for observed traits [7-9]. Thus, we hypothesized that development of novel methods to study existing GWAS datasets would provide additional insight and identify 
new candidate genes. Identify candidate Causal SNPs and Pathways (ICSNPathway) analysis was developed to identify SNPs candidate and their corresponding candidate pathways using GWAS data together with linkage disequilibrium (LD) analysis, functional SNP annotation, and pathway-based analysis (PBA) [10].

We applied ICSNPathway analysis to a glioma GWAS dataset to identify candidate SNPs and mechanisms of glioma susceptibility, and to generate SNP-to-gene-to-pathway hypotheses.

\section{Materials and methods}

Study population. We used a publicly available glioma GWAS dataset from the National Center for Biotechnology Information (NCBI) dbGap (http://www.ncbi.nlm.nih.gov/ projects/gap/cgi-bin/study.cgi?study_id=phs000652.v1.p1), which included the genotypes of 524,589 SNPs obtained from the Human610_Quadv1_B, Human660W-Quad_v1_A, and HumanHap550v3.0 platforms. The dataset comprised 1,856 glioma patients and 4,955 controls of European descent (studies with more than $80 \%$ European-ancestry subjects) from 14 cohort studies belonging to the Cohort Consortium, 3 case-control studies, and 1 population-based case-only study [3]. Cases were newly diagnosed glioma [ICDO-3 codes $9380-9480$ or equivalent], and controls were healthy individuals. The dataset was filtered to remove SNPs showing significant $(\mathrm{p}<0.001)$ Hardy-Weinberg violation, and a call rate cut-off value of $<98 \%$ was used to reduce the impact of genotyping errors. In all, 509,345 SNPs passed the quality control filters.

Identification of candidate causal SNPs and pathways. ICSNPathway analysis was carried out in 2 stages [10]. The first stage involved the pre-selection of candidate causal SNPs by linkage disequilibrium (LD) analysis and functional SNP annotation based on the most significant SNPs. The second stage involved annotating the biological mechanisms underlying pre-selected candidate causal SNPs using the PBA algorithm, $i$-GSEA (improved gene-set enrichment analysis). A full list of glioma GWAS SNP p-values was entered into the ICSNPathway analysis. One concept utilized in the ICSNPathway analysis is LD analysis, which searches the GWAS dataset for the most significant SNPs in LD to identify additional candidate causal SNPs based on an extended dataset, including HapMap data [11]. The other method involves the use of functional SNPs. ICSNPathway analysis pre-selects candidate causal SNPs based on functional SNPs, which are important factors in the underlying genetics of human health. Functional SNPs are defined as SNPs that alter protein or gene expression or the role of a protein in the context of a pathway. They include deleterious and non-deleterious non-synonymous SNPs, SNPs that cause the gain or loss of a stop codon, those resulting in a frameshift, and those located in essential splice sites or regulatory regions. The ICSNPathway server applies the $i$-GSEA PBA algorithm to the full list of GWAS SNP p-values to detect the pathways associated with individual traits. Briefly, the process is as follows. (i) Each SNP is mapped to its nearest gene according to their respective loci in the Ensembl 61 database (http://www.wnsembl.org/biomart/martview), and the maximum SNP $t=-\log (p$-value) values mapped to genes are assigned to represent those genes. Then, all genes are ranked by decreasing representative $t$ values. (ii) For each pathway $S$, the enrichment score (ES, i.e., a Kolmogorov-Smirnov-like running-sum statistic with weight [a]) is calculated, which measures the tendency for genes of a pathway to be located at the top of the ranked gene list. (iii) The $E S$ is then converted to a significant proportion-based $E S$ (SPES) by multiplying the $E S$ by $m_{1} / m_{2}$, where $m_{1}$ is the proportion of significant genes for the pathway $S$ (defined as genes mapped with at least one SHLP among the top 5\% of the most significant SNPs in the GWAS), and $m_{2}$ is the proportion of significant genes for all genes in the GWAS. (iv) SNP label permutation and normalization are employed to generate the distribution of SPESs and to correct for gene variation (bias caused by different genes with different numbers of mapped SNPs) and pathway variation (bias due to different pathways with different numbers of genes). (v) Based on the distribution of SPES values generated by the permutation, a nominal p-value is calculated, and a false discovery rate (FDR) is computed for multiple testing correction.

The phrase "the most significant SNPs" refers to SNPs with a p-value below a certain threshold, which can be specified from the GWAS SNP p-values. The ICSNPathway was used to analyze significant pathways from the original GWAS data when we chose the p-value threshold $\left(<1 \times 10^{-3}\right)$ used in the study. Two parameters were set for the analysis. The first was "within gene," meaning that only p-values of SNPs located within genes were used in the PBA algorithm. The second was an FDR cut-off (0.05) for multiple testing corrections. Control of the FDR is preferred for large-scale testing. Defined as the expected proportion of false-positives among all significant tests, it allows researchers to identify a set of "candidate positives," a high proportion of which are likely to be true-positives. The FDR, a permutation-based approach for multiple comparisons, was used to identify statistically significant genes. There were no specific criteria used to select the number of genes; we used a cut-off range of a minimum of 5 and maximum of 100 to avoid very narrow or very broad functional categories. We discarded pathways that contained over 100 genes to avoid stochastic bias and the inclusion of a general biological process. Out of the several options available for pathway annotation, we selected four pathway databases: the Kyoto Encyclopedia of Genes and Genomes (KEGG) [12], BioCarta, gene ontology (GO) biological process [13], and GO molecular function. This ensured comprehensive coverage of pathways and high-quality information for well-defined pathways.

SNAP was developed to identify and annotate nearby SNPs in LD (proxies) by HapMap (http://www.broadinstitute.org/ $\mathrm{mpg} / \mathrm{snap} /$ ). In this study, we used SNAP to: (i) find proxy SNPs, (ii) determine whether SNP proxies were present in 
genes, (iii) resolve whether associations from multiple SNPs represented similar associations, (iv) plot regional views of associations or LD structures, and (v) retrieve annotations for SNPs [14].

\section{Results}

Candidate SNPs and pathways identified from glioma GWASs. Utilizing the 509,345 GWAS SNP p-values as the input and the most significant SNPs $\left(\mathrm{p}<1 \times 10^{-3}\right)$, ICSNPathway analysis identified 6 candidate SNPs, 5 genes, and 9 pathways (Tables 1, 2, and 3; Figure 1). The 6 candidate SNPs were rs667128 $\left(-\log _{10}[p]=4.889\right), \operatorname{rs619381}\left(-\log _{10}[p]=5.083\right)$, $\operatorname{rs} 1033583\left(-\log _{10}[p]=4.337\right), \operatorname{rs} 2232580\left(-\log _{10}[p]=3.309\right)$, rs4644 $\left(-\log _{10}[p]=4.703\right)$, and $\mathrm{rs} 4652\left(-\log _{10}[p]=4.703\right)$. Four of the 6 candidate SNPs (excluding rs4644 and rs4652) were not in LD with any other SNP. SNP rs4644, which was in LD with rs8007614 $\left(\mathrm{r}^{2}=0.853\right)$, was not represented in the original GWAS dataset. Similarly, rs4652 was not represented in the original GWAS, and was in LD with rs8007614 $\left(\mathrm{r}^{2}=\right.$ $0.96)$. The biological mechanisms suggest that the candidate SNPs may alter the role of their corresponding gene or protein in the context of pathway-associated traits. The 6 candidate SNPs were associated with 9 candidate pathways, having roles in 5 hypothetical biological mechanisms. The hypothetical mechanisms, beginning with the strongest, can be summarized as follows: (i) rs667128 (regulatory region) alters the role of TAS2R8 in taste receptor activity and taste transduction pathways $(p<0.001$, FDR $<0.001 ; p=0.001$, FDR $=0.012)$, (ii) rs619381(nonsynonymous coding (deleterious)) modulates the effect of TAS2R7 on taste receptor activity and taste transduction $(p<0.001, \mathrm{FDR}<0.001 ; p=0.001, \mathrm{FDR}=0.012)$. It has

A

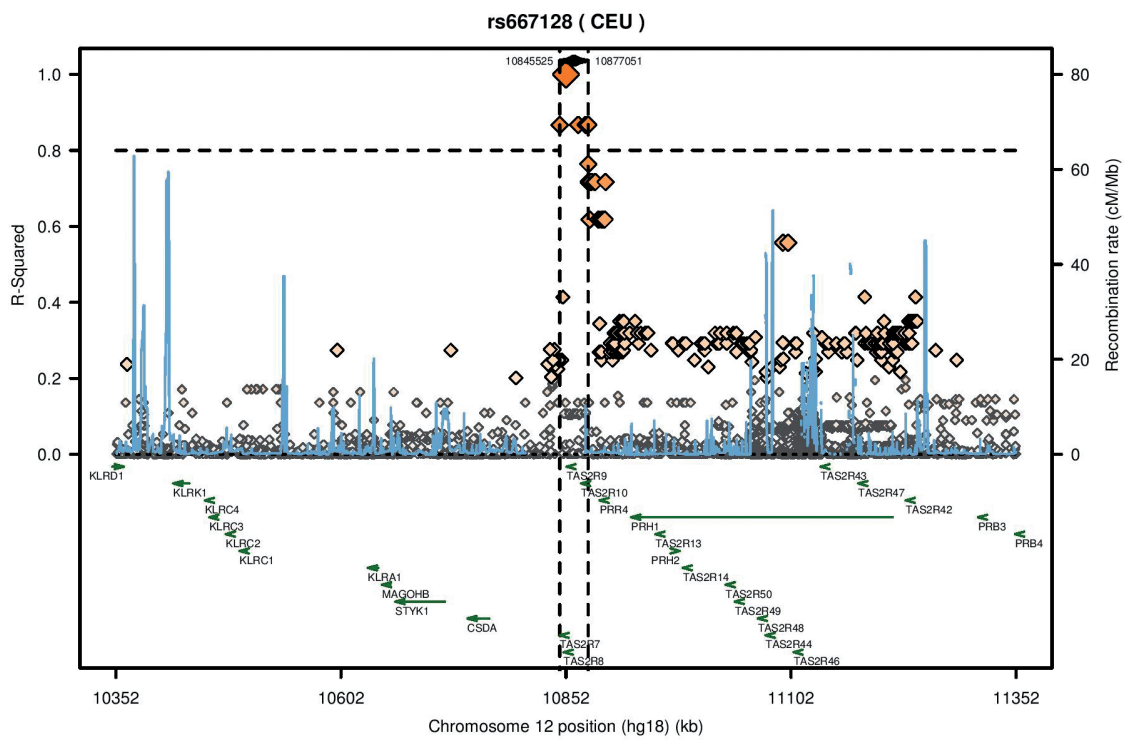

B

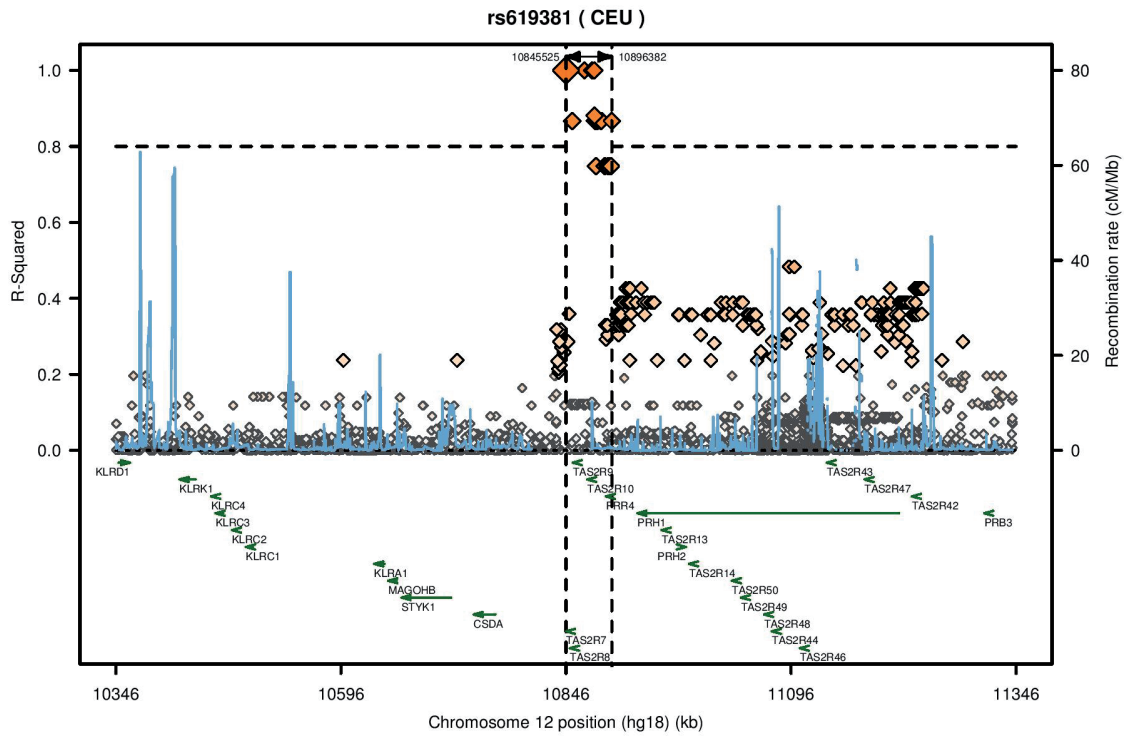


C

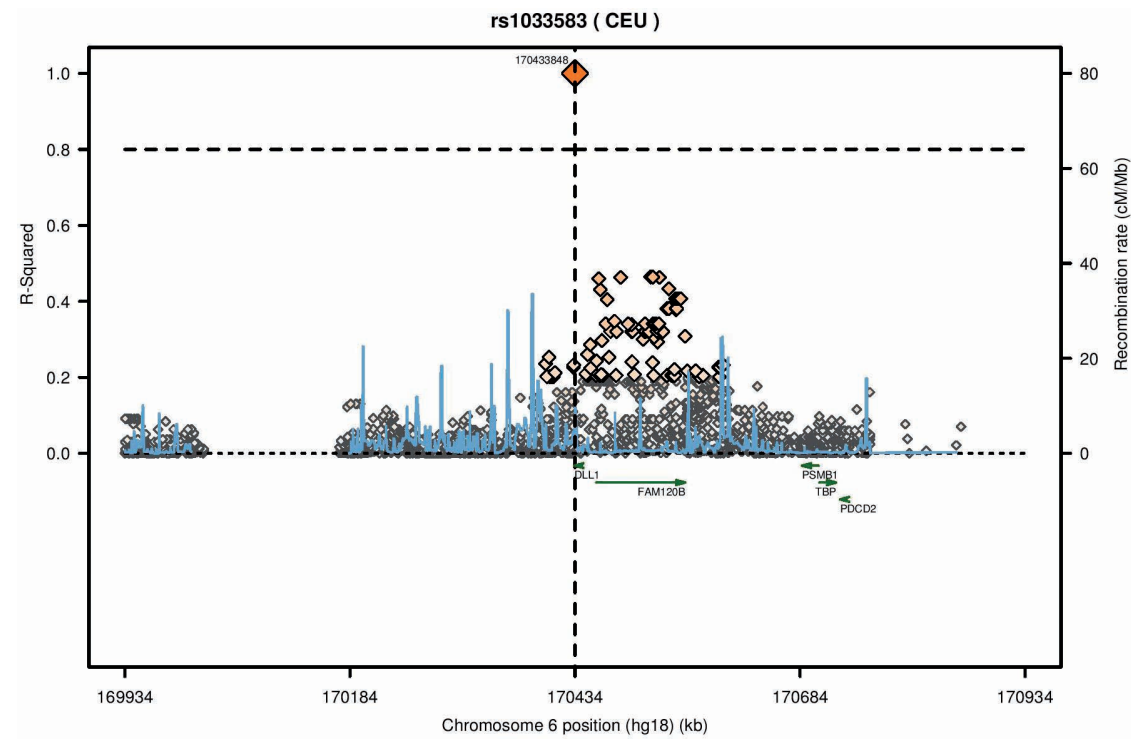

D

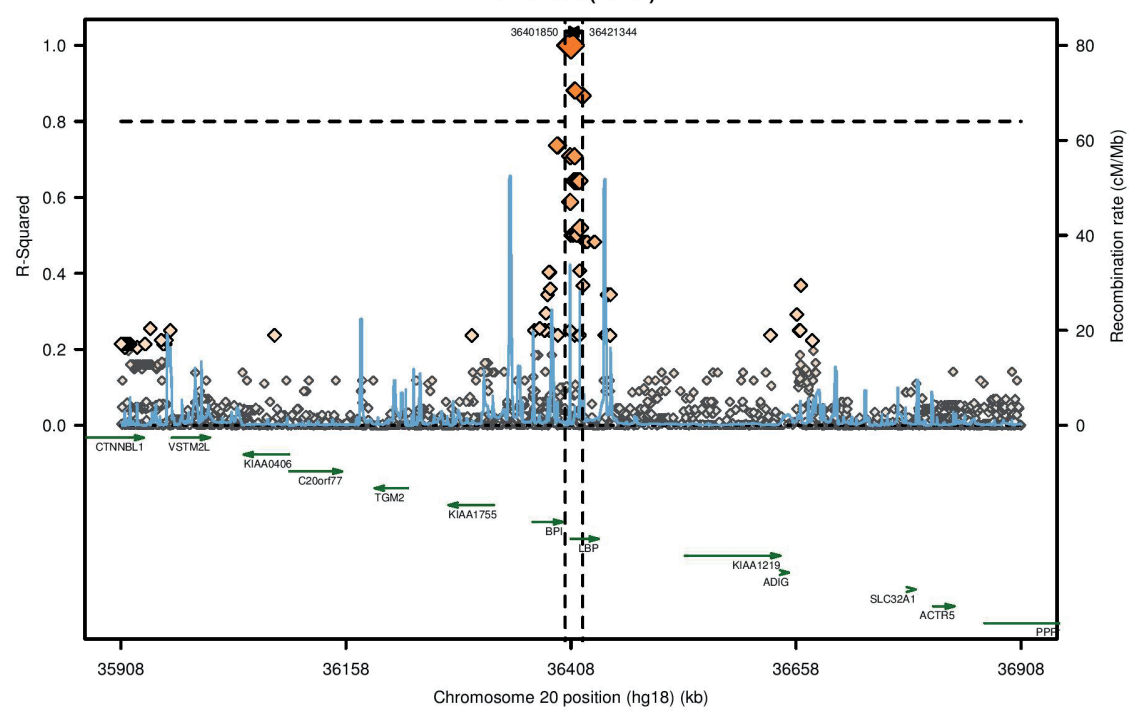

$\mathbf{E}$

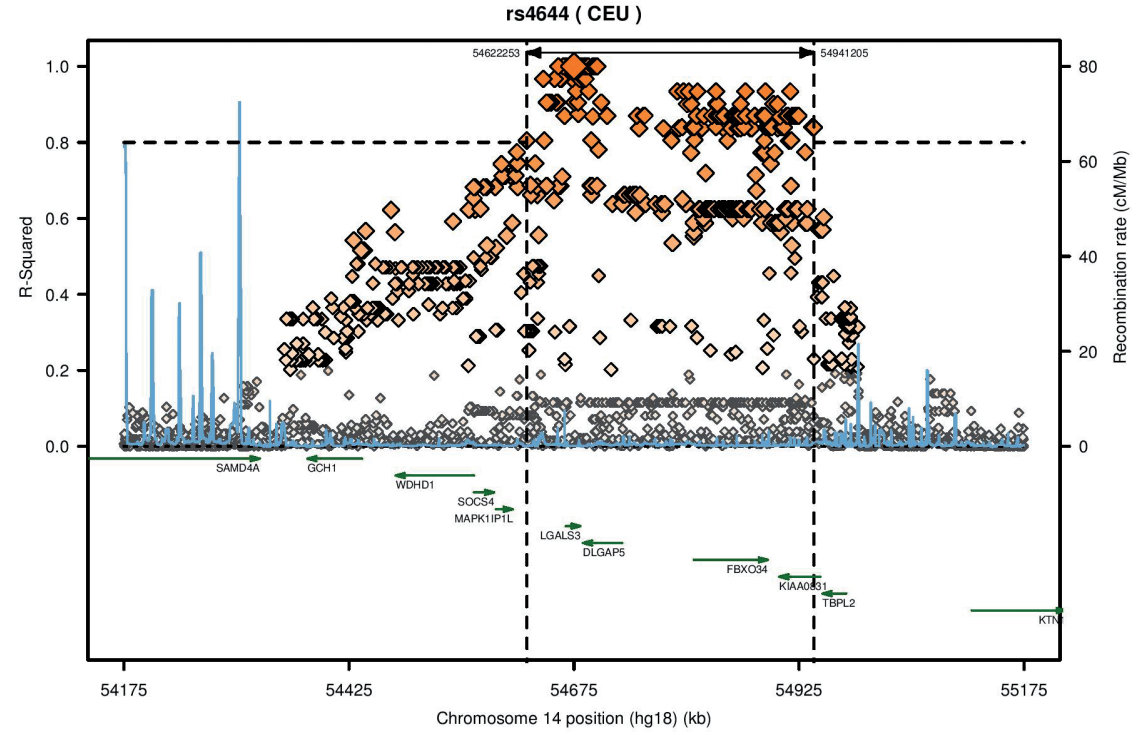

Figure 1 Regional linkage disequilibrium (LD) plots of rs667128 (TAS2R8) (A), rs619381 (TAS2R7) (B), rs1033583 (DLL1) (C), rs2232580 (LBP) (D), and rs4644 (LGALS3) (E) SNPs. SNPs are plotted along with their proxies (based on 1000 Genomes pilot $1 \mathrm{CEU}$ ) as a function of genomic location, and are annotated by the recombination rate across the locus (light blue line). On the y-axis, pairwise $r^{2}$ values are provided for each proxy SNP using color codes. 


\section{Table 1. Candidate SNPs}

\begin{tabular}{|c|c|c|c|c|c|c|c|c|}
\hline Candidate SNP & Functional class & Gene & $\begin{array}{l}\text { Candidate } \\
\text { pathway }\end{array}$ & $-\log _{10}(p)^{\dagger}$ & In LD with & $r^{2}$ & $D^{\prime}$ & $-\log _{10}(p)^{t}$ \\
\hline rs667128 & Regulatory region & TAS2R8 & 13 & 4.889 & rs667128 & - & - & 4.889 \\
\hline rs619381 & Nonsynonymous coding (deleterious) & TAS2R7 & 13 & 5.083 & rs619381 & - & - & 5.083 \\
\hline rs1033583 & Regulatory region & DLL1 & 28 & 4.337 & rs1033583 & - & - & 4.337 \\
\hline rs2232580 & Nonsynonymous coding & $L B P$ & 4567 & 3.309 & rs2232580 & - & - & 3.309 \\
\hline rs 4644 & Nonsynonymous coding (deleterious) & $L G A L S 3$ & 9 & - & rs8007614 & 0.853 & 1.0 & 4.703 \\
\hline rs4652 & Nonsynonymous coding & LGALS3 & 9 & - & rs8007614 & 0.96 & 1.0 & 4.703 \\
\hline
\end{tabular}

$S N P$, single-nucleotide polymorphism; $L D$, linkage disequilibrium; 'Numbers indicate the indices of pathways (listed in Table 3 ) ranked by significance (false discovery rate); ${ }^{\dagger} \log _{10}(p)$ values of candidate causal SNPs in the original genome-wide association studies (GWASs) ; “-” denotes that this SNP was not represented in the original GWAS; ${ }^{\ddagger}-\log _{10}(p)$ values of SNPs in LD with candidate causal SNPs in the original GWAS

Table 2. Functional and association study of genes identified by GWAS pathway analysis

\begin{tabular}{|c|c|}
\hline Gene & Function \\
\hline TAS2R8 & $\begin{array}{l}\text { This gene product belongs to the family of candidate taste receptors, which are members of the G-protein-coupled receptor superfamily. These } \\
\text { proteins are specifically expressed in the taste receptor cells of the tongue and palate epithelia. They are organized in the genome in clusters } \\
\text { and are genetically linked to loci that influence bitterness perception in mice and humans. In functional expression studies, they respond to } \\
\text { bitter tastants. This gene maps to the taste receptor gene cluster on chromosome 12p13. }\end{array}$ \\
\hline TAS2R7 & $\begin{array}{l}\text { Gustducin-coupled receptor implicated in the perception of bitter compounds in the oral cavity and the gastrointestinal tract. Signals through } \\
\text { phospholipase C, beta } 2 \text { (PLCB2) and the calcium-regulated transient receptor potential cation channel, subfamily M, member } 5 \text { (TRPM5). }\end{array}$ \\
\hline DLL1 & $\begin{array}{l}\text { DLL1 is a human homolog of the Notch Delta ligand and is a member of the delta/serrate/jagged family. It plays a role in mediating cell fate } \\
\text { decisions during hematopoiesis. It may play a role in cell-to-cell communication. It acts as a ligand for Notch receptors, and blocks the dif- } \\
\text { ferentiation of progenitor cells into the B-cell lineage while promoting the emergence of a population of cells with T-cell/NK-cell precursor } \\
\text { characteristics. }\end{array}$ \\
\hline$L B P$ & $\begin{array}{l}\text { The protein encoded by this gene is involved in the acute-phase immunologic response to gram-negative bacterial infections. Gram-negative } \\
\text { bacteria contain a glycolipid, lipopolysaccharide (LPS), on their outer cell wall. Together with bactericidal permeability-increasing protein (BPI), } \\
\text { the encoded protein binds LPS and interacts with the CD14 receptor, probably playing a role in regulating LPS-dependent monocyte responses. } \\
\text { Studies in mice suggest that the encoded protein is necessary for the rapid acute-phase response to LPS but not for the clearance of LPS from } \\
\text { circulation. This protein is part of a family of structurally and functionally related proteins, including BPI, plasma cholesteryl ester transfer } \\
\text { protein (CETP), and phospholipid transfer protein (PLTP). It binds to the lipid A moiety of bacterial lipopolysaccharides (LPS), a glycolipid } \\
\text { present in the outer membrane of all Gram-negative bacteria, and acts as an affinity enhancer for CD14, facilitating its association with LPS. }\end{array}$ \\
\hline LGALS3 & $\begin{array}{l}\text { This gene encodes a member of the galectin family of carbohydrate-binding proteins. Members of this protein family have an affinity for beta- } \\
\text { galactosides. The encoded protein is characterized by an } \mathrm{N} \text {-terminal proline-rich tandem repeat domain and a single } \mathrm{C} \text {-terminal carbohydrate } \\
\text { recognition domain. This protein can self-associate through the } \mathrm{N} \text {-terminal domain, allowing it to bind to multivalent saccharide ligands. It } \\
\text { localizes to the extracellular matrix, the cytoplasm and the nucleus and plays roles in numerous cellular functions including apoptosis, innate } \\
\text { immunity, cell adhesion and T-cell regulation. Alternate splicing results in multiple transcript variants. }\end{array}$ \\
\hline
\end{tabular}

been suggested that sweet taste receptors might play important roles in the tumorigenesis in the nervous system [15]. (iii) rs 1033583 (regulatory region) modulates $D L L 1$, regulating cell adhesion and segment specification $(p<0.001, \mathrm{FDR}=0.011$; $p=0.001, \mathrm{FDR}=0.032$ ), (iv) rs2232580 (nonsynonymous coding) affects the role of $L B P$ in the response to lipopolysaccharide, positive regulation of interleukin- 6 production, acute inflammatory response, and in macrophage activation $(0.002$ $<p<0.013 ; 0.012<$ FDR $<0.030$ ), and (v) rs4644 (nonsynonymous coding (deleterious)) and rs4652 (nonsynonymous coding) regulate $L G A L S 3$, affecting immunoglobulin binding $(p=0.010 ; \mathrm{FDR}=0.040)$.

Using a pathway-based approach, we next investigated which genes played roles in the identified pathways and found a distinct clustering of genes involved in the candidate glioma-causal pathways. The most significant pathway was the taste receptor activity pathway, specifically involving TAS2R7, TAS2R14, TAS2R9, TAS2R13, TAS1R2, TAS1R1, and TAS2R16 $(p<0.05)$. The second most significant pathway was that regulating cell adhesion, which involved cyclin-dependent kinase inhibitor 2A (CDKN2A), delta-like protein 1 (DLL1), cadherin 13 ( $C D H 13)$, a disintegrin and metalloproteinase domain-containing protein (ADAM10), transglutaminase 2 (TGM2), GTP binding protein 4 (GTPBP4), kininogen 1 (KNG1), azurocidin 1 (AZU1), arachidonate 12-lipoxygenase (ALOX12), neurofibromatosis type 2 (NF2), Rho GDP dissociation inhibitor (GDI) beta (ARHGDIB), tuberous sclerosis 1 (TSC1), endomucin (EMCN), phosphatase and tensin homolog $(P T E N)$, transforming growth factor, beta-induced (TGFBI), neurofibromatosis type I (NF1), cluster of differentiation 47 (CD47), cyclin-dependent kinase 6 (CDK6), beta-1,4-N-acetyl-galactosaminyl transferase 2 (B4GALNT2), 
Table 3. Candidate causal pathways

\begin{tabular}{|c|c|c|c|c|}
\hline Index & Candidate pathway & Description & $p$ & FDR \\
\hline 1 & TASTE RECEPTOR ACTIVITY & $\begin{array}{l}\text { GO:0008527. Combining with soluble compounds to initiate a change in cell } \\
\text { activity. These receptors are responsible for the sense of taste. }\end{array}$ & $<0.001$ & $<0.001$ \\
\hline 2 & REGULATION OF CELL ADHESION & $\begin{array}{l}\text { GO:0030155. Any process that modulates the frequency, rate or extent of attach- } \\
\text { ment of a cell to another cell or to the extracellular matrix. }\end{array}$ & $<0.001$ & 0.011 \\
\hline 3 & hsa04742 & Taste transduction Taste transduction & 0.001 & 0.012 \\
\hline 4 & RESPONSE TO LIPOPOLYSACCHARIDE & $\begin{array}{l}\text { GO: } 0032496 \text {. A change in state or activity of an organism (in terms of movement, } \\
\text { secretion, enzyme production, gene expression, etc.) as a result of a lipopolysac- } \\
\text { charide stimulus; lipopolysaccharide is a major component of the cell wall of } \\
\text { gram-negative bacteria. }\end{array}$ & 0.002 & 0.012 \\
\hline 5 & $\begin{array}{l}\text { POSITIVE REGULATION OF } \\
\text { INTERLEUKIN-6 PRODUCTION }\end{array}$ & $\begin{array}{l}\text { GO:0032755. Any process that activates or increases the frequency, rate, or extent } \\
\text { of interleukin- } 6 \text { production. }\end{array}$ & 0.013 & 0.027 \\
\hline 6 & ACUTE INFLAMMATORY RESPONSE & $\begin{array}{l}\text { GO:0002526. Inflammation comprising a rapid, short-lived, relatively uniform } \\
\text { response to acute injury or antigenic challenge and characterized by the accumula- } \\
\text { tion of fluid, plasma proteins, and granulocytic leukocytes. An acute inflammatory } \\
\text { response occurs within a matter of minutes or hours, and either resolves within } \\
\text { a few days or becomes a chronic inflammatory response. }\end{array}$ & 0.013 & 0.028 \\
\hline 7 & MACROPHAGE ACTIVATION & $\begin{array}{l}\text { GO: } 0042116 \text {. A change in the morphology and behavior of a macrophage resulting } \\
\text { from exposure to a cytokine, chemokine, cellular ligand, or soluble factor. }\end{array}$ & 0.007 & 0.030 \\
\hline 8 & SEGMENT SPECIFICATION & $\begin{array}{l}\text { GO:0007379. The process whereby segments assume individual identities; exempli- } \\
\text { fied in insects by the actions of the products of the homeotic genes. }\end{array}$ & 0.001 & 0.033 \\
\hline 9 & IMMUNOGLOBULIN BINDING & $\begin{array}{l}\text { GO:0019865. Interacting selectively and non-covalently with an immunoglobu- } \\
\text { lin. }\end{array}$ & 0.010 & 0.040 \\
\hline
\end{tabular}

FDR: false discovery rate

signal-regulatory protein gamma (SIRPG), RAS p21 protein activator (GTPase activating protein) 1 (RASA1), Rho GDP dissociation inhibitor (GDI) gamma (ARHGDIG), Rho family GTPase 1 (RND1), interleukin 12B (natural killer cell stimulatory factor 2 , cytotoxic lymphocyte maturation factor 2, p40) (IL12B), activin A receptor type II-like 1 (ACVRL1), interleukin 18 (interferon-gamma-inducing factor) (IL18), and chemokine (C-X3-C motif) ligand 1 (CX3CL1) $(p<$ 0.05 ). The third pathway was taste transduction. Previous study revealed that sweet taste receptors might play important roles in the tumorigenesis in the nervous system [15]. The fourth pathway was response to LPS. LPS induces nitric oxide (NO) in rat glioma C6 [16], and the study suggested NO derived from inflammation may contribute to the progression of carcinogenesis [16]. The sixth pathway was acute inflammatory response. It is well known that inflammation plays important roles in the pathogenesis of brain tumor [17]. The seventh pathway was macrophage activation, which leads to change in the morphology and behavior of a macrophage resulting from exposure to a cytokine, chemokine, or cellular ligand, and plays a key role in the immune and inflammatory responses. The eighth pathway was segment specification, which assumes individual identities and is exemplified in insects by the actions of the products of the homeotic genes. The ninth pathway was immunoglobulin binding, which interacts selectively and non-covalently with an immunoglobulin. The pathways of segment specification and immunoglobulin binding are involved in the immune system. Glioma development and progression is dependent on evading the immune system by mediating immunosuppression and immune evasion. Lack of recruitment of naïve effector immune cells accounts for most of the immune suppression mediated by glioma cells [15]. Genes without a known immunological function are of particular interest because of their potential to lead to the identification of novel mechanisms for the susceptibility to glioma. This analysis suggests that the pathways and genes identified by PBA may contribute to patient susceptibility to glioma.

\section{Discussion}

Various cellular pathways involving complex molecular networks may play key roles in the development of glioma [18]. If a specific pathway was relevant to disease susceptibility, association signals would be expected to be overrepresented for SNPs in that pathway [4,19-21]. Given the limited power of GWAS to detect single SNP associations, we adopted a pathway-based approach to take into account the biological interplay between genes and to provide insight into how multiple genes might contribute to the pathogenesis of glioma [22-24].

In the present study, we used ICSNPathway analysis to identify 6 candidate SNPs, 5 genes, and 9 pathways, which provided 5 hypothetical biological mechanisms. The most significant SNP-to-gene-to-effect hypothesis was that of rs667128 altering the role of TAS2R8 in taste receptor activity and taste transduction pathways. This gene product is a candidate taste receptor belonging to the G-protein-coupled 
receptor superfamily. These proteins are specifically expressed in the taste receptor cells of the tongue and palate epithelia [25]. The second strongest mechanism was one wherein rs619381 modulates TAS2R7-mediated effects in pathways of taste receptor activity and taste transduction. TAS2R7 plays a role as a G (Gustducin)-coupled receptor and is implicated in the perception of bitter compounds in the oral cavity and gastrointestinal tract [26]. Taste receptor cells in the taste buds transmit gustatory information to the nervous system. Glucose is an essential substrate for brain oxidative metabolism [27]. Brain glucosensors are specialized neurons, modulating their mean firing rate according to changes in glucose concentration [28]. Sweet taste receptors and G-protein-coupled receptor are proposed to be associated with brain glucose sensor [15] Thus, taste-like signaling mechanisms in the brain might be involved in the central regulation of homeostatic processes. A study showed increased expression of sweet taste receptors in C6 rat glioma, suggesting sweet taste receptors might play important role in the tumorigenesis in the mammalian nervous system [15]. The third mechanism identified in this analysis was the modulation of DLL1 by rs1033583 in the regulation of cell adhesion and segment specification. DLL1 acts as a ligand for Notch receptors and blocks the differentiation of progenitor cells into the B-cell lineage while promoting the emergence of a population of cells with T-cell/natural killercell precursor characteristics [29]. It plays a role in mediating cell fate decisions during hematopoiesis and might also play a role in cell-to-cell communication. DLL1 is overexpressed in glioma cell lines and primary human gliomas, and downregulation of $D L L 1$ by RNA interference inhibits proliferation and induces apoptosis in multiple glioma cell lines [30]. The fourth mechanism was that rs2232580 affects the role of $L B P$ in the response to lipopolysaccharide (LPS), positive regulation of interleukin- 6 production, acute inflammatory response, and in macrophage activation. Monocytes and neutrophils required for the innate immune response against LPS and lipoarabinomannan (LAM) express CD14 on their surfaces. LBP acts as an affinity enhancer by disaggregating and catalytically transferring LPS to CD14 [31]. In addition, the CD14-dependent LAM response is greatly facilitated by the addition of LBP [32]. LBP is involved in the acute-phase immunologic response to gram-negative bacterial infections. LPS induces nitric oxide (NO) in rat glioma C6, suggesting NO derived from inflammation may contribute to the progression of carcinogenesis [16]. Inflammatory responses may play a key role in the pathogenesis of brain tumor [17]. The fifth mechanism was that rs4644 and rs4652 regulate LGALS3, affecting immunoglobulin binding. This gene encodes a member of the galectin family of carbohydrate-binding proteins and plays roles in numerous cellular functions including apoptosis, innate immunity, cell adhesion, and T-cell regulation [33]. In the nucleus, LGALS3 acts as a pre-mRNA splicing factor, and is involved in acute inflammatory responses including neutrophil activation and adhesion, chemoattraction of monocytes and macrophages, opsonization of apoptotic neutrophils, and activation of mast cells [33]. LGALS3 accelerates macrophage infiltration and angiogenesis in tumors [34], and is known to be increasingly activated in microglia and macrophages as glioma progresses [35]. There is increasing evidence that the development of malignant disorders is related to complex pathways involving the immune system and inflammatory response [36, 37]. Our analysis, which identified 5 candidate genes that could contribute to glioma risk (TAS2R8, TAS2R7, DLL1, LBP, and $L G A L S 3$ ), uncovered promising glioma-associated genes and pathways related to the immune system and inflammatory responseAlthough additional studies are needed to confirm the association between glioma and these SNPs, genes, and pathways, pathway-based approaches play a complementary role in the identification of novel genes conferring disease susceptibility. Therefore, the results obtained in the present study may lead to the formulation of novel hypotheses for future investigation.

It is well known that most causative genetic alterations in human gliomas involve in either activation of tyrosine kinase pathway or disruption of cell cycle arrest pathways, such as EGFR, PDGFR, p53, p16, p19, cyclin D [2, 3]. However, we did not identify those pathways using our approach. There are two possible explanations on the difference. First, the candidate genes have been identified by univariate analyses of individual SNPs, such as GWAS. GWAS focuses the SNPs with the highest statistical significance, and the genes identified by GWAS account for only a small portion of the heritability of diseases. Testing of each SNP is not well suited for detecting multiple variants with small effects. However, glioma is caused by multiple genetic factors interacting with environmental factors and complex molecular network and pathways play key roles in the susceptibility of glioma. ICSNPathway is used to identify disease-related SNPs, genes, and pathways by interpreting the full list of GWAS SNPs, instead of the most significant SNPs, and searching a large pathway databases. ICSNPathway considers not only the strong association signal of most significant SNPs, but also the combined effect of modest SNPs, which ensures a comprehensive analysis, and play a complementary role in the identification of novel genes that confer disease susceptibility. Second, ICSNPathway is not intended to be used to predict true causal SNPs and pathways because of the limited understanding of genetic basis of complex diseases. The outputs of ICSNPathway indicate possible candidate SNPs and pathways. Thus, we cannot rule out the possibility that our results may be false negative. Our findings suggest that relationships exist between glioma and the genes and pathways identified, but it is needed to validate our approach to identify glioma-associated genes and pathways. ICSNPathway is a novel and validated approach to identify causative genes of human diseases. ICSNPathway approach has been successfully applied to identify causative genes of other diseases such as rheumatoid arthritis [10].

In summary, we examined a glioma GWAS dataset to identify genetic associations with glioma at both the SNP and pathway levels. By applying ICSNPathway analysis to the 
glioma GWAS dataset, we identified 6 candidate SNPs, 5 genes (TAS2R8, TAS2R7, DLL1, LBP, and LGALS3), 9 pathways, and 5 biological mechanisms that may contribute to glioma susceptibility. However, further studies are needed to confirm and explore the genetic variations of the molecular pathways that might be associated with glioma.

Acknowledgements: The authors gratefully acknowledge investigators, for sharing their valuable GWAS data.

\section{References}

[1] GOODENBERGER ML, JENKINS RB GENETICS OF ADULT GLIOMA. Cancer Genet 2012; 205: 613-621. http:// dx.doi.org/10.1016/j.cancergen.2012.10.009

[2] MELIN B, JENKINS R GENETICS IN GLIOMA: LESSONS LEARNED FROM GENOME-WIDE ASSOCIATION STUDIES. Curr Opin Neurol 2013; 26: 688-692. http://dx.doi. org/10.1097/WCO.0000000000000033

[3] RAJARAMAN P, MELIN BS, WANG Z, MCKEAN-COWDIN R, MICHAUD DS et al. Genome-wide association study of glioma and meta-analysis. Hum Genet 2012; 131: 1877-1888. http://dx.doi.org/10.1007/s00439-012-1212-0

[4] MANOLIO TA Genomewide association studies and assessment of the risk of disease. N Engl J Med 2010; 363: 166-176. http://dx.doi.org/10.1056/NEJMra0905980

[5] JOHNSON AD, O'DONNELL CJ An open access database of genome-wide association results. BMC Med Genet 2009; 10: 6. http://dx.doi.org/10.1186/1471-2350-10-6

[6] SRIDHAR S, AL-MOALLEM B, KAMAL H, TERRILE $M$, STALLINGS RL New insights into the genetics of neuroblastoma. Mol Diagn Ther 2013; 17: 63-69. http://dx.doi. org/10.1007/s40291-013-0019-6

[7] WANG K, LI M, HAKONARSON H Analysing biological pathways in genome-wide association studies. Nat Rev Genet 2010; 11: 843-854. http://dx.doi.org/10.1038/nrg2884

[8] LEE YH, SONG GG Pathway analysis of genome-wide association studies on uric acid concentrations. Hum Immunol 2012; 73: 805-810. http://dx.doi.org/10.1016/j. humimm.2012.05.004

[9] LEE YH, BAE SC, CHOI SJ, JI JD, SONG GG Genome-wide pathway analysis of genome-wide association studies on systemic lupus erythematosus and rheumatoid arthritis. Mol Biol Rep 2012; 39: 10627-10635. http://dx.doi.org/10.1007/ s11033-012-1952-X

[10] ZHANG K, CHANG S, CUI S, GUO L, ZHANG L et al. ICSNPathway: identify candidate causal SNPs and pathways from genome-wide association study by one analytical framework. Nucleic Acids Res 2011; 39: W437-443. http://dx.doi. org/10.1093/nar/gkr391

[11] INTERNATIONAL HAPMAP C, ALTSHULER DM, GIBBS RA, PELTONEN L, ALTSHULER DM et al. Integrating common and rare genetic variation in diverse human populations. Nature 2010; 467: 52-58. http://dx.doi.org/10.1038/nature09298

[12] KANEHISA M, GOTO S, FURUMICHI M, TANABE M, HIRAKAWA M KEGG for representation and analysis of molecular networks involving diseases and drugs. Nucleic Acids Res 2010; 38: D355-360. http://dx.doi.org/10.1093/nar/ gkp896

[13] ASHBURNER M, BALL CA, BLAKE JA, BOTSTEIN D, BUTLER $\mathrm{H}$ et al. Gene ontology: tool for the unification of biology. The Gene Ontology Consortium. Nat Genet 2000; 25: 25-29. http://dx.doi.org/10.1038/75556

[14] JOHNSON AD, HANDSAKER RE, PULIT SL, NIZZARI MM, O'DONNELL CJ et al. SNAP: a web-based tool for identification and annotation of proxy SNPs using HapMap. Bioinformatics 2008; 24: 2938-2939. http://dx.doi. org/10.1093/bioinformatics/btn564

[15] YI C Sweet taste receptors in normal and pathological rat brain [Dissertation]. University of Tübingen, 2011. Available from: http://nbn-resolving.de/urn:nbn:de:bsz:21-opus-57722.

[16] CHEN TJ, SHEN SC, LIN HY, CHIEN LL, CHEN YC Lipopolysaccharide enhancement of 12-o-tetradecanoylphorbol 13-acetate-mediated transformation in rat glioma C6, accompanied by induction of inducible nitric oxide synthase. Toxicol Lett 2004; 147: 1-13. http://dx.doi.org/10.1016/j. toxlet.2003.10.012

[17] SOWERS JL, JOHNSON KM, CONRAD C, PATTERSON JT, SOWERS LC The role of inflammation in brain cancer. Adv Exp Med Biol 2014; 816: 75-105. http://dx.doi. org/10.1007/978-3-0348-0837-8 4

[18] ZHAO Z, TAN X, ZHAO A, ZHU L, YIN B et al. microRNA-214-mediated UBC9 expression in glioma. BMB Rep 2012; 45: 641-646. http://dx.doi.org/10.5483/ BMBRep.2012.45.11.097

[19] CHOI SJ, RHO YH, JI JD, SONG GG, LEE YH Genome scan meta-analysis of rheumatoid arthritis. Rheumatology (Oxford) 2006; 45: 166-170. http://dx.doi.org/10.1093/rheumatology/kei128

[20] LEE YH, NATH SK Systemic lupus erythematosus susceptibility loci defined by genome scan meta-analysis. Hum Genet 2005; 118: 434-443. http://dx.doi.org/10.1007/s00439-0050073-1

[21] FRIDLEY BL, BIERNACKA JM Gene set analysis of SNP data: benefits, challenges, and future directions. Eur J Hum Genet 2011; 19: 837-843. http://dx.doi.org/10.1038/ ejhg. 2011.57

[22] ZEGGINI E, IOANNIDIS JP Meta-analysis in genome-wide association studies. Pharmacogenomics 2009; 10: 191-201. http://dx.doi.org/10.2217/14622416.10.2.191

[23] ELBERS CC, VAN EIJK KR, FRANKE L, MULDER F, VAN DER SCHOUW YT et al. Using genome-wide pathway analysis to unravel the etiology of complex diseases. Genet Epidemiol 2009; 33: 419-431. http://dx.doi.org/10.1002/ gepi.20395

[24] PEDROSO I, BREEN G Gene set analysis and network analysis for genome-wide association studies. Cold Spring Harb Protoc $2011 ; 2011$.

[25] UENO Y, SAKURAI T, OKADA S, ABE K, MISAKA T Human bitter taste receptors hTAS2R8 and hTAS2R39 with differential functions to recognize bitter peptides. Biosci Biotechnol Biochem 2011; 75: 1188-1190. http://dx.doi. org/10.1271/bbb. 100893 
[26] CHANDRASHEKAR J, MUELLER KL, HOON MA, ADLER E, FENG $L$ et al. T2Rs function as bitter taste receptors. Cell 2000; 100: 703-711. http://dx.doi.org/10.1016/S0092-8674(00)80706-0

[27] TSACOPOUlos M, MAgistretTi PJ Metabolic coupling between glia and neurons. J Neurosci 1996; 16: 877-885.

[28] GONZ LEZ JA, JENSEN LT, FUGGER L, BURDAKOV D Metabolism-independent sugar sensing in central orexin neurons. Diabetes 2008; 57: 2569-2576. http://dx.doi.org/10.2337/ $\underline{\mathrm{db} 08-0548}$

[29] ANDRAWES MB, XU X, LIU H, FICARRO SB, MARTO JA et al. Intrinsic selectivity of Notch 1 for Delta-like 4 over Delta-like 1. J Biol Chem 2013; 288: 25477-25489. http:// dx.doi.org/10.1074/jbc.M113.454850

[30] QIAN CF, YAN W, ZHANG JX, SHI L, QIAN J et al. Notch1 induces enhanced expression of Delta-like-1 in the U251MG glioma cell line. Int J Mol Med 2009; 24: 445-451.

[31] ECKERT JK, KIM YJ, KIM JI, G RTLER K, OH DY et al. The crystal structure of lipopolysaccharide binding protein reveals the location of a frequent mutation that impairs innate immunity. Immunity 2013; 39: 647-660. http://dx.doi. org/10.1016/j.immuni.2013.09.005
[32] ORR SL, TOBIAS P LPS and LAM activation of the U373 astrocytoma cell line: differential requirement for CD14. J Endotoxin Res 2000; 6: 215-222. http://dx.doi. org/10.1179/096805100101532072

[33] CARLSSON MC, BENGTSON P, CUCAK H, LEFFLER H Galectin-3 guides intracellular trafficking of some human serotransferrin glycoforms. J Biol Chem 2013; 288: 28398 28408. http://dx.doi.org/10.1074/jbc.M113.487793

[34] JIA W, KIDOYA H, YAMAKAWA D, NAITO H, TAKAKURA N Galectin-3 accelerates M2 macrophage infiltration and angiogenesis in tumors. Am J Pathol 2013; 182: 1821-1831. http://dx.doi.org/10.1016/j.ajpath.2013.01.017

[35] BINH NH, SATOH K, KOBAYASHI K, TAKAMATSU M, HATANO Y et al. Galectin-3 in preneoplastic lesions of glioma. J Neurooncol 2013; 111: 123-132. http://dx.doi.org/10.1007/ s11060-012-1005-2

[36] MOHME M, NEIDERT MC, REGLI L, WELLER M, MARTIN R Immunological challenges for peptide-based immunotherapy in glioblastoma. Cancer Treat Rev 2014; 40: 248-258. http://dx.doi.org/10.1016/j.ctrv.2013.08.008

[37] PARNEY IF, HAO C, PETRUK KC Glioma immunology and immunotherapy. Neurosurgery 2000; 46: 778-791; discussion 791-792. 